\title{
Beetroot and carrot juice increase the risk of thyroid nodules and hypothyroidism in breast cancer patients
}

\author{
Student Diana Viorela ARTENE ${ }^{1}, \mathrm{MD}, \mathrm{MNS}$; Cristian I. BORDEA², MD, PhD; \\ Mircea D. CROITORU ${ }^{3}$, PhD; Prof. Alexandru BLIDARU ${ }^{1,2}, \mathrm{MD}, \mathrm{PhD}$ \\ ${ }^{1}$ „Carol Davila“ University of Medicine and Pharmacy, Bucharest, Romania \\ ${ }_{2}^{2}$,Prof. Dr. Al. Trestioreanu“ Institute of Oncology, Bucharest, Romania \\ ${ }^{3}$ University of Medicine and Pharmacy, Targu Mures, Romania
}

\begin{abstract}
After diagnosis, many breast cancer patients start consuming high quantities of beetroot and carrot juice, in the hope of improving the outcome of their treatment. However, these foods are very high in nitrate, which can competitively inhibit the uptake of iodine by the thyroid, potentially leading to hypothyroidism or thyroid nodules. We applied a nitrate and iodine food frequency questionnare (asking about dairy, fish, seafood and iodized salt for iodine intake and spinach, carrots, beetroot, lettuce and arugula for nitrate intake) to 353 $E R+/ P R \pm / H E R 2-l u m i n a l ~ A$ and $B$ breast cancer patients during antiestrogenic treatment. We excluded patients with a thyroid disease diagnosis prior to the cancer diagnosis, smokers, ex-smokers and those with renal disease or bipolar disorder.

The only correlations found between dietary intake of iodine and nitrate and the incidence of de novo thyroid nodules were: decreased risk for a daily intake of minimum $250 \mathrm{ml}$ dairy; and increased risk for daily intakes of over $200 \mathrm{~g}$ spinach, $250 \mathrm{~g}$ carrots or $250 \mathrm{~g}$ beetroot.

The correlations between dietary intake of nitrate and the incidence of de novo hypothyroidism were: decreased risk for a daily intake of iodized salt $2.5 \mathrm{~g}$ and minimum of $100 \mathrm{~g}$ fish or $250 \mathrm{ml}$ dairy; and increased risk for daily intakes of over $250 \mathrm{~g}$ carrots or $250 \mathrm{~g}$ beetroot.

The results of this study support the hypothesis that the increased intake of nitrate-rich foods - particularly beetroot and carrot juice - is a risk factor for de novo hypothyroidism or thyroid nodules, after a breast cancer diagnosis.
\end{abstract}

Keywords: breast cancer, thyroid disease, beetroot juice, competitive iodine inhibition by nitrate, $\mathrm{Na}+/ \mathrm{I}-$ symporter

\section{INTRODUCTION}

lodine bioavailability is directly influenced by $\mathrm{Na}^{+} / \mathrm{I}^{-}$symporter functionality. But this is not only simport thyroid level, but also in the salivary glands, gastric mucosa, kidney, placenta, ovary and mammary glands ut also in the salivary glands, kidneys, ovaries and mamary glands (1).

Studies show that optimal functioning of the $\mathrm{Na}^{+} / \mathrm{I}^{-}$symporter is particularly important for women's health, through preventing fibro-cystic dysplasia and hyperplasia of the mammary gland. Based on their findings, Kilbane et al proposed the introduce the $\mathrm{Na}^{+} / \mathrm{I}^{-}$symporter dysfunction among breast cancer etiological mechanisms, as they proved that both in fibroadenoma and in malignant cells, breast iodine is very low (2).

Moreover, IGF- 1 and TGF- $\beta$ inhibit the transmembrane expression of the $\mathrm{Na}^{+} / \mathrm{I}^{-}$symporter, further argument that supports the relationship between breast cancer and breast atypia and this symporter functionality (3).

lodine deficiency - either by low iodine intake or by excessive intake of nitrate that may

Corresponding author:

Mircea Dumitru Croitoru, University of Medicine and Pharmacy, 38 Gheorghe Marinescu Street, 540139, Tirgu Mures, Romania

E-mail: croitoru.mircea@umftgm.ro 
competitively inhibit the uptake of iodine by the thyroid gland - is common in both developed and in developing countries.

Most healthy people do not consume excessive quantities of nitrate rich foods (such as beets, carrots, spinach, cabbage, lettuce or arugula), but many breast cancer patients change their diets after their diagnosis - and the main food they believe will help them towards curing their cancer is the freshly squeezed juice of carrots and beetroot - beet juice representing the nitrate source of choice in the majority of studies done on nitrate's health impact, due to its average content of $1.5 \mathrm{~g}$ nitrate per $\mathrm{Kg}$ of beetroot.

The anticancer effect of beetroot juice is based on its high antioxidant content (betanine, isobetaine and ferulic acid esters) (4) - but breast cancer patients either completely ignore, or do not know that beet does not only contain nitrate, while scientists look for denitrification ways for this food (5).

Firstly, even though antioxidants are important in preventing de novo tumorigenesis, current scientific literature does not support that fact that the excessive intake of food or dietary supplements high in antioxidants leads to a better cancer prognosis (6). For instance, even though betanine has a cytotoxic potential comparable to that of doxorubicin (7), antioxidant intake during chemotherapy is descuraged by most oncologists, based on studies that prove that antioxidants' protective action extends to both healthy cells and malign cells, thus decreasing adjuvant treatments efficacy (8).

Secondly, malign cells uses glucose as their main nutrient, and beet is very high in glucose, which can be readily used by such type of cells to survive and multiply (9).

Consequently, if we take into account not only its antioxidant content, but also the nitrate and glucose ones, beetroot ceases to be the universal panacea considered by most cancer patients.

For healthy women, the main consequences of iodine deficiency are thyroid hypertrophy and the emergence of single or multiple nodules. Additionally, as iodine contributes to the maintenance of normal estrogen blood levels, through stimulating the secretion of estriol and by decreasing the secretion of estrone and estradiol (10) - iodine deficiency increases the risk of fibrocystic breast disesase, ER+/PR \pm /HER \pm breast cancers, ovarian cancer, hyperestrogenism, and obesity with gynoid adyposity (11).
For ER+/PR $\pm / H E R \pm$ breast cancer patients, in addition to the risks mentioned above, iodine deficiency further contributes to:

- Decreased sensitivity for testosterone-a mechanism involved in sarcopenia during chemotherapy and osteosarcopenia during antiestrogenic treatment - both increasing the risk of sarcopenic obesity, which, in turn, indirectly increases the risk of recurrence or de novo carcinogenesis.

- Increased sensitivity of estrogen - a mechanism that can decrease antiestrogenic treatment efficacy, along with an posing an increased risk of endometrial hyperplasia.

Based on the aformentioned facts, this study aims to answer two questions:

1. Is there any correlation between the occurrence of thyroid disease after breast cancer dtiagnosis, and patients' weight gain during the oncological treatment?

2. Is there any correlation between the occurrence of thyroid disease, after breast cancer diagnosis, and the intake of foods high in iodine and nitrate?

\section{METHODS}

The study was conducted between December 2015 and February 2016, at the Department of Surgical Oncology II of „Prof. Dr. Al. Trestioreanu“ Institute of Oncology, Bucharest, Romania, with the approval of the Ethics Committee of the Institute.

The study has been conducted in accordance with the ethical standards laid out in the 1964 Declaration of Helsinki and its later amendments. We declare no conflict of interest.

We selected 353 patients that complied with our study inclusion criteria: $E R+/ P R \pm /$ HER2-breast cancer patients under antiestrogenic treatment. We excluded smokers and ex-smokers, those with triple negative or HER2+ tumors, metastases, recurrences, de novo carcinogenesis, and those kidney disease or bipolar disorder treated with lithium.

We used a food frequency questionnaire to asses dietary intake of iodine and nitrate and we analysed the results in SPSS, using a Fisher exact test for establishing the relevance of statistical correlations $(p<0,05)$ between the intake of foods high in iodine and nitrate, considering the immunohistochemical diagnosis and the antiestrogenic treatment received by the 
patients (Tamoxifen, Anastozole, Letrozole or Exemestane).

\section{Food frequency questionnaire}

Date:

Name:

Age:

Height:

Waist circumference:

Weight:

Immunohistochemical diagnosis:

Endocrinological diagnosis:

Oncology treatment stage:

Comorbidity:

Medication:

Food supplements:

Weight gain during treatment:

1. Do you consume iodized salt?

- Yes - how much iodized salt do you consume per day:

- No - how much non-iodized salt do you consume per day:

2. Do you eat fish?

- Yes:

- What quantity do you eat per serving:

- How often do you eat that amount:

- I do not eat fish.

3. Do you eat seafood?

- Yes:

- What quantity do you eat per serving:

- How often do you eat that amount:

- I do not eat seafood.

4. Do you eat dairy (milk, yogurt, buttermilk, kefir)?

- Yes:

- What quantity do you eat per serving:

- How often do you eat that amount:

- I do not eat dairy.

5. Do you eat cruciferous vegetables (white cabbage, red cabbage, Brussels sprouts)?

- Yes:

- What quantity do you eat per serving:

- How often do you eat that amount:

- I do not eat cabbage.

\section{Do you eat lettuce?}

- Yes:

- What quantity do you eat per serving:

- How often do you eat that amount:

- I do not eat lettuce.

\section{Do you eat arungula?}

- Yes:

- What quantity do you eat per serving:

- How often do you eat that amount:

- I do not eat arungula.

\section{Do you eat spinach?}

- Yes:

- What quantity do you eat per serving:

- How often do you eat that amount:

- I do not eat spinach.

9. Do you eat carrots?

- Yes:

- What quantity do you eat per serving:

- How often do you eat that amount:

- I do not eat carrots.

\section{Do you eat beetroot?}

- Yes:

- What quantity do you eat per serving:

- How often do you eat that amount:

- I do not eat beetroot.

\section{RESULTS AND DISCUSSION}

The current study supports what is already known in the scientific literature studying the connection between breast cancer and thyroid disease: aproximatly a quarter of patients develop de novo thyroid disease after the breast cancer diagnosis.

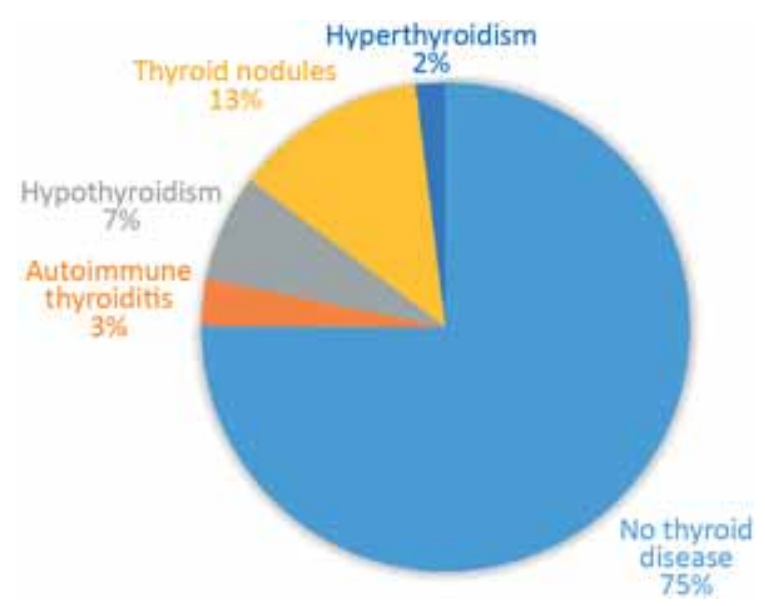

FIGURE 1. De novo thyroid disease incidence after breast cancer diagnosis

The current study proves a direct correlation between patients $\mathrm{BMI}$ and de novo thyroid disease occurred after breast cancer diagnosis. 


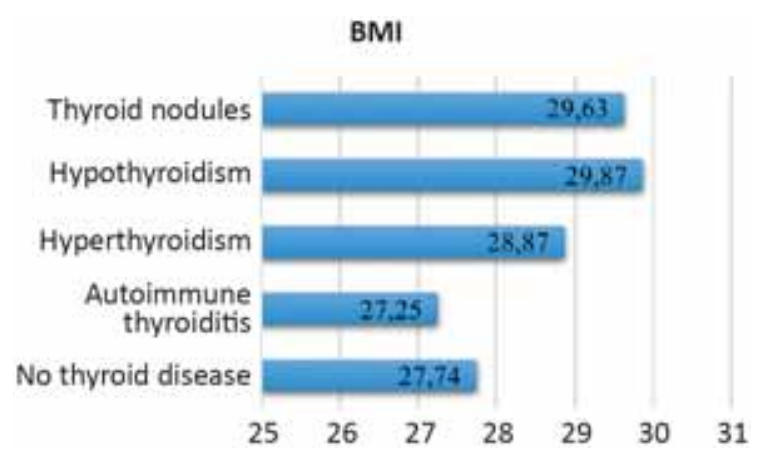

FIGURE 2. Comparative BMI during breast cancer treatment correlated with de novo thyroid disease

Weight gain during breast cancer treatment worsens prognosis, increasing recurrence and de novo carcinogenesis risks. In our study, patients diagnosed with thyroid nodules and hypothyroidism also had the greatest weight gain.

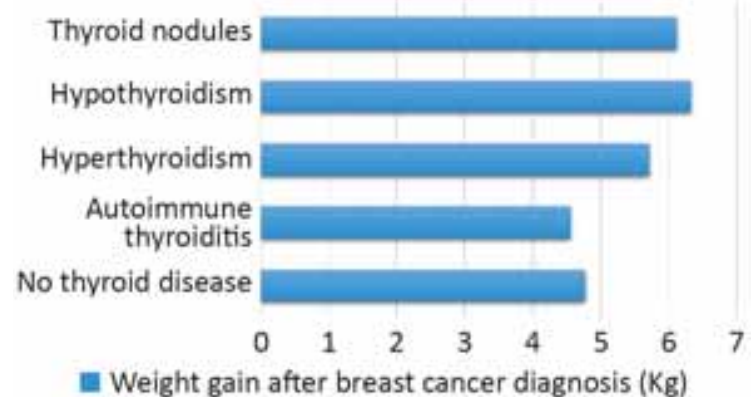

FIGURE 3. Comparative weight gain during breast cancer treatment correlated with de novo thyroid disease

To establish the correlation between the intake of foods high in iodine and nitrate and de novo thyroid disease diagnosis in breast cancer patients, the questionnaires were analyzed using SPSS, Fisher exact test $(p<0.05)$.
Spearman correlation coefficient ( $r S$ ) can range between -1 and $1,-1$ signifying a negative correlation (representing protection against thyroid disease, after breast cancer diagnosis) and +1 positive correlation (representing risk factor for thyroid disease, after breast cancer diagnosis).

Since an 0 value of $\mathrm{rS}$ indicates no correlation, $\mathrm{rS}$ values very close to 0 indicate no statistical correlation.

In our study, the only correlations between dietary intake of foods high in nitrate and:

- De novo thyroid nodules after breast cancer diagnosis - were: protection by a minimum intake of $250 \mathrm{ml}$ dairy/day; and an increased risk by consuming more than $200 \mathrm{~g}$ spinach, $250 \mathrm{~g}$ carrots and $250 \mathrm{~g}$ beetroot/day.

- De novo thypothyroidism after breast cancer diagnosis - were: protection by a minimum intake of $2.5 \mathrm{~g}$ iodized salt, $100 \mathrm{~g}$ fish and 250g dairy/ day; and an increased risk by consuming more than $250 \mathrm{~g}$ carrots and $250 \mathrm{~g}$ beetroot/day.

Due to the small number of patients with hyperthyroidism or autoimmune thyroiditis, interpretation of results in these two types of thyroid pathology had no statistical significance, thus requiring further studies, which will utilize the appropriate number of breast cancer patients with those thyroid pathologies (Table 1).

Among the questionned breast cancer patients, dairy consumption was inversely associated with the risk of developing hypothyroidism and thyroid nodules.

TABLE 1. Comparative Fisher Exact Test results between food intake and de novo thyroid disease diagnosis in breast cancer patients

\begin{tabular}{|c|c|c|c|c|c|c|c|c|c|c|}
\hline \multirow{2}{*}{ Food/day } & \multicolumn{2}{|c|}{ Content } & \multicolumn{2}{c|}{ Thyroid Nodules } & \multicolumn{2}{c|}{ Hypothyroidism } & \multicolumn{2}{c|}{ Hyperthyroidism } & \multicolumn{2}{c|}{$\begin{array}{c}\text { Autoimmune } \\
\text { Thyroiditis }\end{array}$} \\
\cline { 2 - 13 } & $\begin{array}{c}\text { lodine } \\
(\mu \mathrm{g})\end{array}$ & $\begin{array}{c}\text { Nitrate } \\
(\mathrm{g})\end{array}$ & $\mathrm{rS}$ & $\mathrm{p}$ & $\mathrm{rS}$ & $\mathrm{p}$ & $\mathrm{rS}$ & $\mathrm{p}$ & $\mathrm{rS}$ & $\mathrm{p}$ \\
\hline $\begin{array}{c}2.5 \mathrm{~g} \text { iodized } \\
\text { salt }\end{array}$ & 50 & - & -0.07 & 0.6025 & -0.56 & $0.0082^{*}$ & -0.57 & 0.0820 & 0.39 & 0.4194 \\
\hline 100g fish & 200 & - & -0.08 & 0.7275 & -0.67 & $0.0024^{*}$ & -0.22 & 0.7177 & 0 & 1.0000 \\
\hline $250 \mathrm{ml}$ dairy & 125 & - & -0.38 & $0.0137^{*}$ & -0.64 & $0.0015^{*}$ & -0.64 & 0.0831 & 0.21 & 0.7139 \\
\hline $200 \mathrm{~g}$ lettuce & - & 0.8 & 0.08 & 0.6933 & -0.13 & 0.5573 & 0.40 & 0.6000 & 0.15 & 0.8028 \\
\hline $\begin{array}{c}200 \mathrm{~g} \\
\text { spinach }\end{array}$ & - & 0.4 & 0.40 & $0.0189^{*}$ & 0.20 & 0.4256 & 0.31 & 0.6838 & - & - \\
\hline $250 \mathrm{~g}$ carrots & - & 0.2 & 0.75 & $0.0001^{*}$ & 0.66 & $0.0011^{*}$ & 0.35 & 0.3888 & -0.67 & 0.1361 \\
\hline $\begin{array}{c}250 \mathrm{~g} \\
\text { beetroot }\end{array}$ & - & 0.4 & 0.74 & $0.0001^{*}$ & 0.46 & $0.0302^{*}$ & 0.40 & 0.6000 & -0.37 & 0.4972 \\
\hline $\begin{array}{c}200 \mathrm{~g} \\
\text { cabbage }\end{array}$ & - & 0.15 & 0.09 & 0.5217 & 0.27 & 0.2102 & 0.27 & 0.2102 & -0.19 & 0.4693 \\
\hline
\end{tabular}




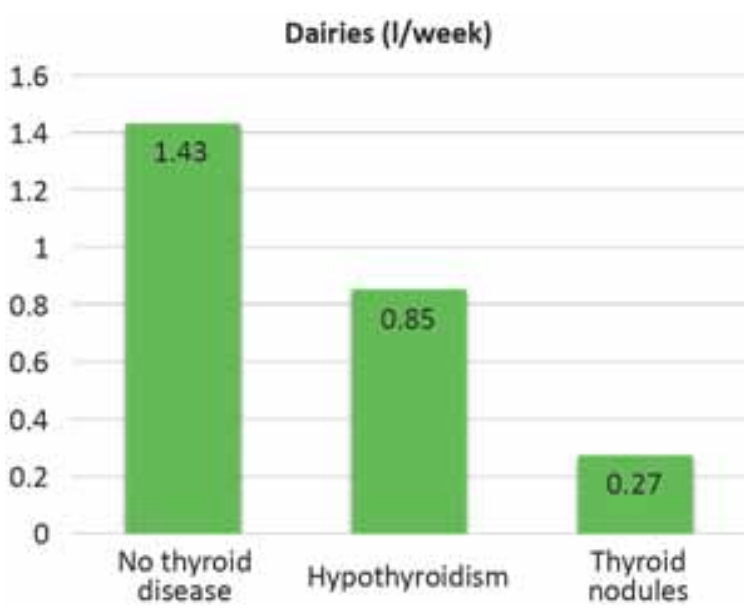

FIGURE 4. Comparative dairy intake in patients without thyroid disease or with thyroid nodules or hypothyroidism

In the questionned breast cancer patients, a daily consumption of $2.5 \mathrm{~g}$ iodized salt is protective against thyroid nodules and hypothyroidism. Most patients with thyroid nodules are not consuming too little salt, but non-iodized salt.

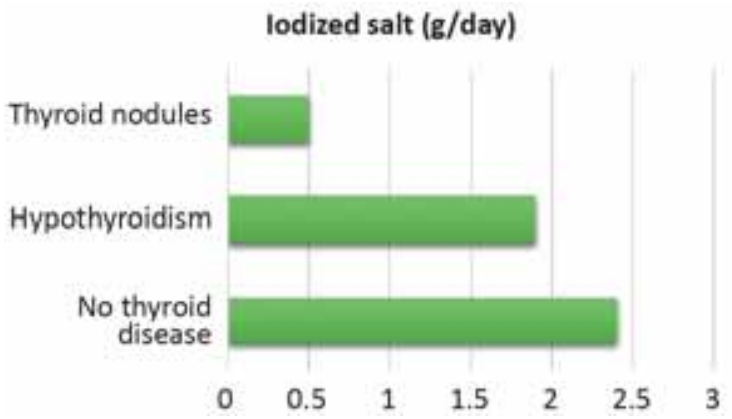

FIGURE 5. Comparative iodized-salt intake in patients without thyroid disease or with thyroid nodules or hypothyroidism

In the questionned breast cancer patients, we found a minimal difference between the fish intake of patients without thyroid disease and that of those with hypothyroidism, however, the fish intake of patients with thyroid nodules was deemed insufficient.

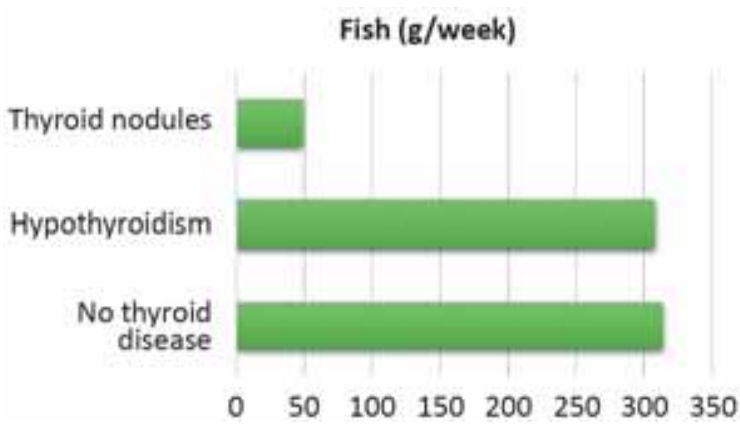

FIGURE 6. Comparative fish intake in patients without thyroid disease or with thyroid nodules or hypothyroidism
Despite representing annual minimal intake - because the study was performed during winter months - carrot and beetroot consumption above the limits presented in Table 1 is a risk factor for de novo thyroid nodules and hypothyroidism, after the diagnosis of breast cancer.

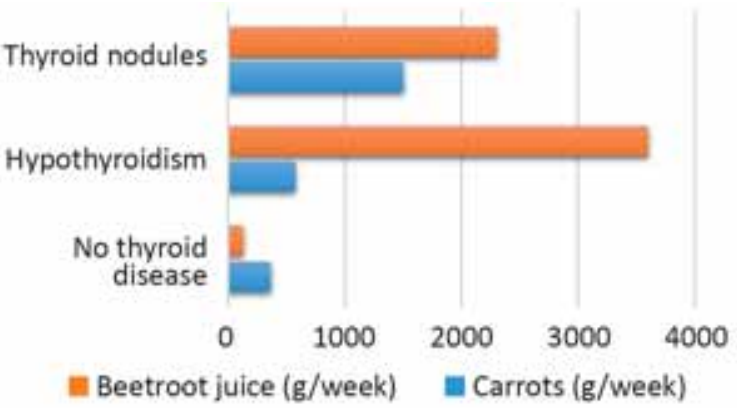

FIGURE 7. Comparative beetroot and carrot intake in patients without thyroid disease or with thyroid nodules or hypothyroidism

\section{STUDY LIMITATIONS}

Due to the fact that most of the questionned patients did not consumed seafood or arungula, we consider the statistic correlation correlation between these foods' intake and de novo thyroid disease, after breast cancer diagnosis, as equivocal.

In addition, because the study was performed between December and February winter months, the food intake and consumption frequency cannot be extrapolated for spring or summer months, while we consider this intake as the minimum intake these patients have during the year.

Also, because this study is based only on patients' answers to the food frequency questionnaire, we need to validate our findings through conductng further studies with toxicology analisys of iodine and nitrate urinary levels.

\section{CONCLUSION}

Our results support the hypothesis that an excessive intake of foods high in nitrate - in particular, beetroot and carrot juice - represents a risk factor for developing de novo thyroid nodules or hypothyroidism, after a breast cancer diagnosis, through the inhibition of iodine uptake through the $\mathrm{Na}^{+} / \mathrm{I}^{-}$symporter by nitrates. 


\section{REFERENCES}

1. Carrasco N. lodide transport in the thyroid gland, Biochim Biophys Acta, 1993, 1154:65-82.

2. Kilbane M.T. et al. Tissue lodine Content and Serum-Mediated $125 \mathrm{I}$ Uptake-Blocking Activity in Breast Cancer 1, The Journal of Clinical Endocrinology \& Metabolism, 2000, 85.3: $1245-1250$.

3. Nicolussi A. et al. TGF- $\beta$ control of rat thyroid follicular cells differentiation, Molecular and cellular endocrinology, 2003, 207.1: 1-11.

4. Kujala T.S. et al. Phenolics and betacyanins in red beetroot (Beta $v$ ulgaris) root: Distribution and effect of cold storage on the content of total phenolics and three individual compounds, Journal of Agricultural and Food Chemistry, 2000, 48.11: 5338-5342.
5. Kolb E. et al. Potential nitrosamine formation and its prevention during biological denitrification of red beet juice, Food and Chemical Toxicology, 1997, 35.2: 219-224.

6. Seifried H.E. et al. The antioxidant conundrum in cancer, Cancer Research, 2003, 63.15: 4295-4298.

7. Kapadia J. et al. Cytotoxic effect of the red beetroot (Beta vulgaris $L$.) extract compared to doxorubicin (Adriamycin) in the human prostate (PC-3) and breast (MCF-7) cancer cell lines, Anti-Cancer Agents in Medicinal Chemistry (Formerly Current Medicinal Chemistry-Anti-Cancer Agents), 2011, 11.3: 280-284.

8. D'Andrea G.M. Use of antioxidants during chemotherapy and radiotherapy should be avoided, CA: a cancer journal for clinicians, 2005, 55.5: 319-321.
9. Greiner E.F., Guppy M., Brand K. Glucose is essential for proliferation and the glycolytic enzyme induction that provokes a transition to glycolytic energy production, Journal of Biological Chemistry, 1994, 269(50): 3148431490.

10. Wright J.V. Bio-identical steroid hormone replacement: selected observations from 23 years of clinical and laboratory practice, Annual of New York Academy of Sciences, 2005, 1057:506-24.

11. Thomas B.S., Bulbrook R.D., Goodman M.J. Thyroid Function and the Incidence of Breast Cancer in Hawaiian, British and Japanese Women, International Journal of Cancer, 1986, 38:325-329. 